\title{
Combined SEM Electron-Beam-Induced Current and Cathodoluminescence Imaging and STEM Structural Analysis of GaN Light Emitting Diodes
}

\author{
C. M. Parish, C. L. Progl, M.E. Salmon, and P. E. Russell
}

Department of Material Science and Engineering, Analytical Instrumentation Facility, North Carolina State University, Campus Box 7531, Raleigh, NC, 27695

Gallium nitride and its alloys ( $\mathrm{In}, \mathrm{Ga}) \mathrm{N}$ and $(\mathrm{Al}, \mathrm{Ga}) \mathrm{N}$ are important optoelectronic materials, and dominate in the green to ultra-violet application range. Unfortunately, GaN and its alloys are currently grown on lattice-mismatched substrates, such as $6 \mathrm{H}-\mathrm{SiC}$ or sapphire. This results in large densities of crystallographic defects within the epilayers. Electron-beam-induced current (EBIC) and cathodoluminescence (CL) are powerful electron-microscope based techniques for the analysis of electronic and optical properties, respectively, of semiconductors. Nitride semiconductor layers and devices have been examined by these techniques previously [1, 2]. However, studies intended to analyze defects, and combined EBIC-CL studies, are lacking.

In this work, a low-cost, custom-designed apparatus for simultaneous CL and EBIC analysis in SEM was implemented. Based around a micro-positioned parabolic mirror and light-collection optics, this apparatus allows for the collection of most of the light emitted by the sample, as well as the collection of the EBIC signal. The system is implemented into a Hitachi S-3200N microscope. The collection optics and feed-through are shown in Fig. 1.

Applications of this system to blue-emitting (In,Ga)N-based LEDs are shown in Figure 2. In Figure 2(a), a secondary-electron image of a portion of an LED sample is shown. Parts (b) and (c) show EBIC and panchromatic CL images, respectively. Fig. 2(b) and (c) clearly show optoelectronic inhomogeneities and defects within the structure of the device, which are entirely missed by the SEM imaging mode. These micrographs indicate that simple SEM imaging is insufficient to characterize any sort of performance of the LED devices; rather, advanced techniques to probe the electron band structure are needed. The EBIC image (Fig. 2(b)) clearly shows two size scales of feature: large dark areas $\sim 5$ microns in diameter, and individual black spots that are much smaller. The individual black spots have a density of $\sim 10^{8} / \mathrm{cm}^{2}$, which is indicative of dislocation densities in GaN-based materials [3]. Further analysis of the EBIC and CL indicates that areas of strong EBIC signal correlate with areas of strong CL signal, and vice-versa. This may imply that dark areas contain defects which reduce the minority carrier lifetime and cause both observed effects.

By performing CL spectroscopy at different accelerating voltages, the depth of penetration of the beam can be varied. Figure 3 shows CL spectra acquired at 10, 20 and 30kV, which correspond to $0.6,2$, and 4 micron electron ranges in GaN. The increase of the yellow-band defect emission $(\sim 560 \mathrm{~nm})$ with increasing $\mathrm{kV}$ implies that the yellow-associated defects are deeper in the epilayers. These defects, as well as those indicated by EBIC and CL imaging, are further explored by FIB-lift out followed by STEM imaging. STEM imaging of a blue LED epilayer shows a highly defected buffer layer and threading dislocations, as well as the quantum well structure.

[1] A. Y. Polyakov et al., Solid State Electronics, 47 (2003), 51.

[2] F. A. Ponce et al., Applied Physics Letters, 68(1) (1996), 57. 
[3] L. Chernyak et al., Solid State Electronics, 45 (2001), 1687.
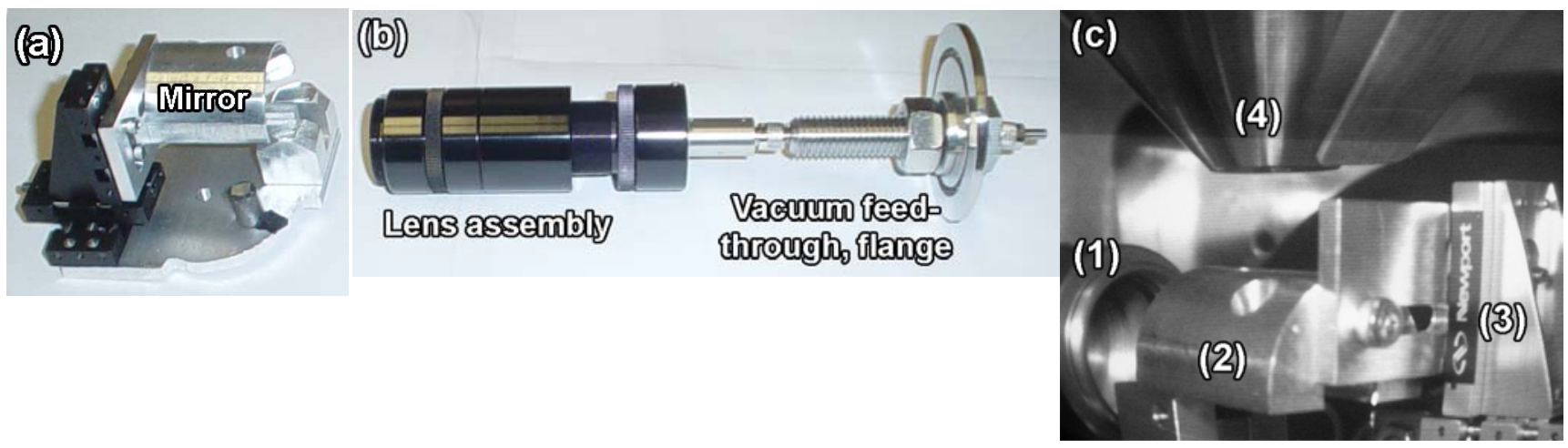

Figure 1: Photograph of the improved CL-EBIC apparatus. (a) Sample holder, featuring a micropositioned parabolic mirror. (b) Lens stack and fiber-optic vacuum feed through and flange. (c) Chamberscope image of the apparatus inside the SEM. The first lens (1), mirror (2), micropositioner (3), and SEM polepiece (4), can all be seen.
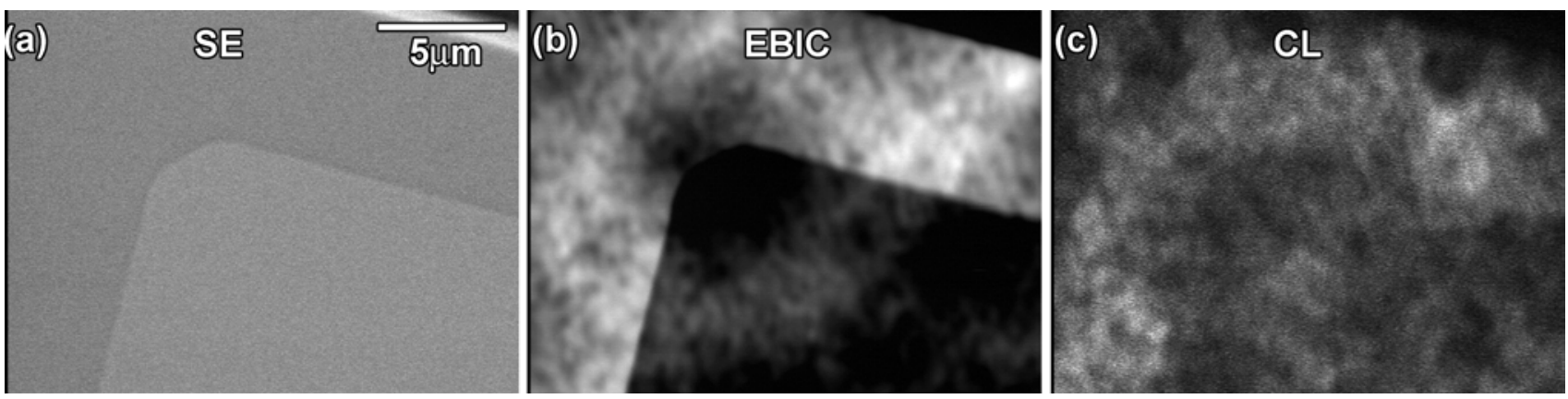

Figure 2: (a) Secondary electron image of a portion of a GaN-based LED; note the smooth surface; the only noticeable feature is the edge of the metallization layer. (b) EBIC image of the same LED, indicating electronic non-uniformity. (c) CL image of the same LED, indicating corresponding optical inhomogeneities.

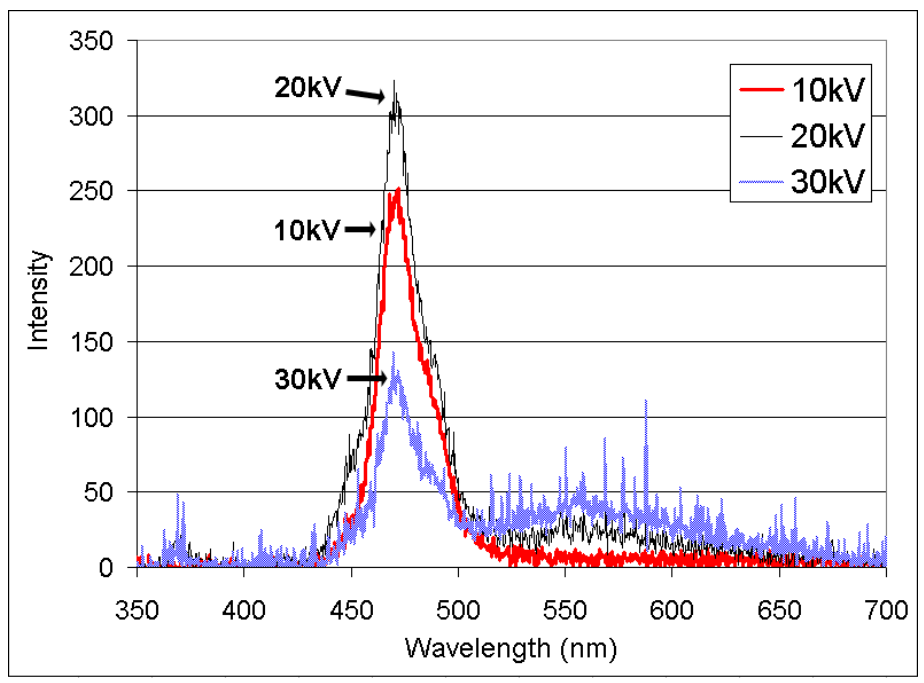

Figure 3: Cathodoluminescence spectroscopy of LED sample under different accelerating voltages. As the voltage increases, the blue QW peak $(\sim 460 \mathrm{~nm})$ increases, then decreases; the UV GaN peak $(\sim 360 \mathrm{~nm})$ and yellowband defect peaks $(\sim 560 \mathrm{~nm})$ both monotonically increase; it is inferred that the yellow-associated defects must be deeper in the epilayer. 